RTIs and immigrant populations has not been well described to date. This work will assess, by systematic review, the relationship between RTI risk and immigrant populations.

Methods Immigrants were defined as those with both parents not native to the country he is residing in. Eligible studies were identified by standard systematic review methodology. Results The initial search yielded 41 articles, only 5 described RTIs specifically among immigrant populations. Swedish data showed no statistically significant difference in RTI risk for young (18-26 years) foreign-born (FB) drivers when compared to native born (NB) drivers. Drivers of all ages showed a 1.5 to 2.5 times increased risk. Greek data showed that immigrant permanent residents have a higher risk for RTI when compared to Greek drivers. Australian data show evidence of higher risk of RTI for pedestrians from a non-English speaking or righthand side driving country. For all other road users, FB were generally at similar or lower risk of dying or being hospitalised due to road trauma than those NB.

Discussion/conclusions There is a surprising paucity of data on the RTI risks in immigrant populations. There are contrasting findings as regards the differential risk for RTI for FB when compared to NB road users. Present research in this field is not commensurate to the population at risk.

\title{
0650 A SYSTEMATIC REVIEW OF ROAD TRAFFIC INJURY RISKS FOR IMMIIGRANT POPULATIONS
}

R J Consunji*, A Jumangit, S Ameratunga Correspondence: Department of Surgery, University of the Philippines, Philippine General Hospital, Taft Avenue, Manila 1100, Philippines

10.1136/ip.2010.029215.650

Introduction There are currently some 200 million migrants and immigrants worldwide, majority of these coming from low and middle-income countries (LMICs), where risks for road traffic injury (RTI) are high. The relationship between 